\title{
A Review of the Research on Symptom Clusters in Cancer Survivors
}

\author{
Gui Li \\ Department of Nephrology/Nursing Department, Peking University First Hospital, Beijing, China \\ Email: 79552566@qq.com
}

How to cite this paper: Li, G. (2021) A Review of the Research on Symptom Clusters in Cancer Survivors. Open Journal of Nursing, 11, 423-434. https://doi.org/10.4236/ojn.2021.116036

Received: May 12, 2021

Accepted: June 8, 2021

Published: June 11, 2021

Copyright (c) 2021 by author(s) and Scientific Research Publishing Inc. This work is licensed under the Creative Commons Attribution International License (CC BY 4.0).

http://creativecommons.org/licenses/by/4.0/ (c) (i) Open Access

\begin{abstract}
Cancer survivors often experience multisymptom clusters, substantial discomfort and lower QOL (quality of life) than the general population. This article summarized the progress of the research on symptom clusters in cancer survivors, and reviewed the definitions, influencing factors, and identified approaches and interventions regarding symptom clusters. The findings allow recommendations to be made in clinical nursing to improve the QOL of cancer survivors. In future studies, longitudinal, large-sample and multicenter studies are recommended to obtain more in-depth information about the changes in symptom and symptom cluster experience perceived by groups of cancer survivors.
\end{abstract}

\section{Keywords}

Symptom Clusters, Cancer Survivors, Symptom Management

\section{Introduction}

A symptom cluster has been described as a set of two or more symptoms that are present at the same time and that are related to each other [1]. All symptoms in a cluster are separate but deeply interrelated, and one symptom can have an influence on another symptom through its impact on a third symptom [2]. Cancer survivors have long experienced multiple symptoms during the period of disease and therapeutic treatment [3]. Some studies have demonstrated that these symptoms are usually present at the same time; thus, most cancer survivors experience 8 or even more symptoms at a time [4] [5]. Definitions of symptom clusters have been provided in previous publications, and some researchers have posed related questions about the potential importance of clustered symptoms; these questions have covered many aspects, such as feasible key factors related to the potential disease, reactions to therapeutic treatment, disease prognosis, and 
quality of life (QOL) [6] [7]. QOL is an outcome to measure how people live [8]. Cancer survivors who undergo related treatment, such as surgery, chemotherapy, and radiotherapy, often experience multiple symptoms, substantial discomfort and low QOL [9]. Therefore, the purpose of this article was to summarize the latest progress in the research on symptom clusters in cancer survivors, and review the definitions, influencing factors, identified approaches and interventions regarding symptom clusters. The findings allow recommendations to be made for clinical nursing to improve the QOL of cancer survivors.

\section{Definitions of Symptom Clusters}

Definitions of a symptom cluster are usually described as a steady aggregation of interrelated symptoms that occur at the same time [10] [11]. While there are still several disagreements regarding some basic components of the concept of symptom clusters. For example, many researchers have their own opinions about the association among symptoms in a single cluster. Some researchers have determined the relations among different symptoms based on correlations [12]. Other researchers have measured the relationships based on the internal impacts of symptoms on the outcomes [13]. A few researchers have also suggested that symptoms could be linked to one another via a universal mechanisms or pathology [14].

Additionally, researchers have disagreed about whether a symptom could be present in several different clusters at the same time. A large number of studies have placed each symptom in only one cluster, but Francoeur [15] found that it was feasible to allow several clusters to have a symptom in common. It is necessary to determine which conception of symptom clusters is being applied to confirm the significance of the relations among symptoms in a cluster.

There is also disagreement in the conception of symptom clusters regarding the lowest number of symptoms that can constitute a cluster. Dodd and colleagues [16] proposed at least 3 symptoms must be present to form a cluster, but Kim's team [17] suggested that only two minimum symptoms are necessary to constitute a cluster. Since their work, a large amount of data-based research has found that the clustering of two symptoms has a negative effect on survivors' QOL or functional status [18], while other researchers have supported the need for at least three symptoms to be considered a cluster [19].

In addition, it remains unclear whether all the symptoms in a cluster must occur simultaneously [20]. These divergences show different opinions about the definition of symptom clusters. Differences in the research design, identification of clusters, features of the research population and sample size may also lead to such inconsistency. Confirming the clinical, practical and theoretical meanings of symptom clusters could answer these questions about the concept of symptom clusters.

In summary, based on the research purpose and questions, clinical practice experience and a previous literature review were combined to define the concept 
of a symptom cluster as a group of two or more symptoms that are presented at the same time and that are interrelated. All symptoms in a cluster are separate but also deeply interrelated, and one symptom can have an influence on another symptom through its effects on a third symptom.

\section{The Content of Symptom Clusters}

\subsection{Influencing Factors of Symptom Clusters}

Studies have shown that certain clinical and demographic variables, for example, age, gender, employment status, comorbid conditions, stage of disease, types of cancer, modality of treatment, trajectory of treatment, time point of measurement, and many other factors, might affect symptom clusters. These factors have all been shown to potentially affect symptom clusters [21].

\subsection{The Influence of Symptom Clusters on Patient Outcomes}

Functional status is affected by symptom clusters. Some data showed that functional status was negatively correlated with the number or intensity of symptom clusters [22] [23] [24]. Miaskowski et al. [25] discovered that survivors reporting a low degree of four symptoms (somnipathy, fatigue, feelings of pain and depression) showed the greatest functional status. A longitudinal study was performed by Dodd et al. [26] to confirm this result. Although the relationship between symptom clusters and functional status was confirmed, the types of symptom clusters that influenced functional status varied in different ways. Additionally, the longitudinal influences of particular symptom clusters on the functional status of cancer survivors remain unclear.

QOL is also affected by symptom clusters. The relationship between QOL and symptom clusters had a similar link as that between functional status and symptom clusters. Some researchers found that survivors with multiple and severe symptom clusters were more likely to have poor QOL [27]. Additionally, one particular cluster, namely, that comprising fatigue and depression, seemed to consistently negatively impact QOL, and explained from $29 \%$ to $42 \%$ of the variance in QOL in various studies [28]. While some studies have shown the influence of other specific clusters on QOL, for instance, physical and emotional clusters, the connection between other clusters and QOL remains unclear [29]. In addition, although a few studies have used longitudinal designs, the time effect of particular symptom clusters on QOL in cancer survivors has not been well established.

Based on the symptom management model, mortality is considered one of the most important outcome indictors in the symptom experience. Gift and colleagues conducted a longitudinal study to explore symptom clusters in 112 survivors with newly diagnosed lung cancer, and they found that death at 6 to 19 months of diagnosis could be predicted not only by patient age and stage of cancer but also by the severity of symptoms at 6 months after diagnosis. A secondary data analysis was performed in the same study, and only physical symp- 
toms were evaluated in the cluster confirmation. Another study of advanced colorectal cancer survivors showed that even though the researchers did not find symptom clusters, mortality was found to be related to multiple symptoms that occurred concurrently with chemotherapy [30]. The results of these studies suggested that multiple symptoms might have an impact on mortality, but the effects of symptom clusters on the death rate remain unclear.

Many studies have found depression as one of the most common symptoms in a cluster [31] [32], while other researchers have investigated the effects of symptom clusters on depression, and explored whether clusters involving malnutrition, general malaise, and gastrointestinal symptoms were independent indicators of depression [33]. Due to the different clustering results and sample sizes in these studies, it is difficult to compare their findings. Additionally, the use of a single-item measurement tool to assess symptoms in one of the studies could pose a threat to the validity of the results. Furthermore, whether depression ought to be explored as a symptom in a cluster or as a result of symptom clustering requires further research.

The findings on the impact of symptom clusters on survivors' outcomes are an important indicator for assessing the nature of symptom cluster studies. Four outcomes were observed in the previous symptom cluster studies. Functional status and QOL were often negatively related to the severity and quantity of symptom clusters. The severity of some symptom clusters indicated the mortality of certain cancer survivors. Depression was found to be an outcome of some symptom clusters. According to the previous studies examined here, the cross-sectional and longitudinal effects and outcomes of cancer survivors' symptoms and symptom clusters have still not been thoroughly investigated. Understanding the effects and outcomes of symptom clusters on cancer survivors could be helpful not only to maintain progress in research on symptom clusters but also to develop targeted interventions for symptom clusters.

\subsection{Measurement of Symptom Clusters}

The C-SAS (Chemotherapy Symptom Assessment Scale) is usually chosen to evaluate the symptoms of chemotherapy. This previously used instrument is patient focused and clinically relevant, and it has been subjected to stringent standard validity, structural validity, test-retest reliability, and internal consistency tests (1/4 0.75) [34]. For every individual symptom, such as nausea, vomiting, fatigue and oral problems, cancer survivors are asked to score the morbidity, severity and related distress to provide detailed information on the overall symptom experience.

The MSAS (Memorial Symptom Assessment Scale) is a validated symptom assessment tool with multiple dimensions that evaluates the severity, frequency, and distress of patients in relation to 32 highly prevalent individual symptoms [35] [36]. According to the introduction to the scale, the MSAS has been used in research with survivors living with ovarian carcinoma [37], breast carcinoma [38], head and neck carcinoma [39], and cancer pain [40]. 
The MDASI (MD Anderson Symptom Inventory) is a patient-report clinical and research instrument that is often used to measure multisymptom outcomes. It is usually applied to assess the severity of symptoms that are experienced by cancer survivors and the interference of these symptoms with daily life. There are 13 core symptoms that have been found to have the highest frequency and/or severity among cancer survivors with various treatment types [41]. The MDASI-GI (MD Anderson Symptom Inventory Gastrointestinal Cancer Module) is a site-specific MDASI module that is focused on gastrointestinal cancer. In addition to 13 core symptom items and 6 interference items from the MDASI, the MDASI-GI also has items evaluating 5 gastrointestinal cancer-specific symptoms: constipation, diarrhea or watery stools, dysphagia, change in taste and bloating. Chen [42] found that the Cronbach's a values of the subscales of the Chinese version of the MDASI-GI were 0.842 and 0.859 .

\subsection{Approaches to Identify Symptom Clusters}

Following the most universal symptomatic approach, researchers have hypothesized that the most general symptoms may have clustered before empirical research. The observation of correlations between symptoms [43], concurrent symptoms [44], mediation and interaction among symptoms [45], and subgroups of cancer survivors with similar symptom cluster profiles [46] are the most common cluster identification methods. Among them, the observation of mediation effects and interaction effects can also be used to identify the content of symptom clusters. Clarifying subgroups of cancer survivors according to clusters might be a convenient approach for translation into clinical practice because of the confirmed similar symptom profiles among cancer survivors. As the outcomes of symptom clusters are mainly based on the selected symptoms in cluster identification, additional efforts should be made in developing basic principles of adding and eliminating symptoms in the identification process.

Even though the most universal symptomatic method provides an approach to comprehend symptom clustering, one important limitation to this method is the question of whether it is adequate to choose only the most general symptoms to identify a cluster. Because the symptoms chosen in identifying cluster directly determine the cluster outcomes, adding or removing any symptoms can change the clustering results. If only the most general symptoms in a cluster identity are chosen without a reasonable theoretical basis, the outcomes of this method may be neither reasonable nor reliable.

In all possible symptom-based methods, any potential symptoms experienced by cancer survivors may be relevant to the confirmation of a cluster, and the clustering of symptoms is identified after statistical analysis. The most common approach is to cluster based on potential factors or components. The other approaches include temporal pattern clustering, central symptom clustering, causal connection clustering, and subgroup clustering [47]. Because symptom clusters confirmed by this method are mainly identified through statistical analysis, the 
clinical significance of symptom cluster outcomes needs to be given more attention. Understanding the clinical significance of each factor might reduce the objectivity of this method, and it may also lead to inconsistency in the cluster outcomes.

\subsection{Symptom Clusters in Cancer Survivors}

A symptom cluster in cancer survivors is explained as two or more symptoms presenting at the same time that are particularly exacerbated during treatments, such as surgery, chemotherapy, radiation, biotherapy, hormonal therapy, and targeted therapy [48]. Tiredness, pain, depression, and sleep disorders are cross-cluster symptoms [49]. Due to the high percentage of cancer survivors who receive chemotherapy, it is reasonable to start a symptom evaluation by assessing the incidence and severity of tiredness, sleep disorders, pain, and depression when the chemotherapy is conducted. Tiredness was found to be related to all types of therapeutic treatment and the symptoms of sleep disorders, pain, and depression [50]. Some studies have noted that pain influences tiredness, sleep disorders, and depression [51].

A study found that the 20 most common symptoms among breast cancer survivors receiving chemotherapy were cough, pain, diarrhea, nausea, vomiting, loss of appetite, astriction, urgency to urinate, urinary burning, hot flashes, rectal irritation, swelling of the arms or legs, shortness of breath, sore throat, dry mouth, skin lesions in the treated area, irritation at the IV site, tiredness, depression, cognitive impairments, and sleep disorders [52]. Several symptom clusters were summarized. For example, the research identified a pain-fatigue-insomnia-depression cluster, a cough-dyspnea cluster, and a nausea-vomiting-diarrhea cluster [53].

Many cancer survivors have to undergo chemotherapy at some point in the cancer treatment process. Chemotherapy blocks the growth of cancer cells in the cell cycle, which determines the process of the growth and development of all cells. Thus, both normal and malignant cells are destroyed when chemotherapy is administrated, leading to side effects and adverse symptoms. Fatigue, pain, sleep disorders, depression, nausea and vomiting, appetite loss, change in taste, feelings of weakness, and oral problems constituted a symptom cluster that was associated with chemotherapy. Fatigue, also described as mental fatigue or physical fatigue, was the most common symptom, affecting more than $96 \%$ of cancer survivors undergoing chemotherapy, and its incidence and severity were associated with the regimen used and dose intensity [54]. In addition, fatigue, pain, sleep disorders, depression, and other chemotherapy-induced toxicities were highly correlated. Chemotherapy has also been found to interact with physical function via its effect on pain, fatigue, and sleep disorders. Chemotherapy can stimulate chemoreceptor trigger zones to cause the symptoms of nausea and vomiting. Alkylating agents usually lead to the symptoms of nausea and vomiting, and cisplatin is the most highly emetogenic agent [55]. Nausea and vomiting could lead to loss of appetite, change in taste, weakness, and fatigue. All of the 
symptoms could be grouped into a potential symptom cluster due to chemotherapy treatment [56]. Oral symptoms of cancer survivors might also be caused by chemotherapy since the oral cavity is a region where cells are rapidly divide, and mucositis can be caused by many agents.

\subsection{Interventions for Symptom Clusters}

Symptom cluster management must be useful in clinical practice. First, interventions should be based on evidence. Second, key resources should be available for evidence-based and consensus-based interventions in the form of clinical practice guidelines. Although it is difficult to develop effective interventions for symptom clusters for different populations, there are still a few researchers making some attempts.

Interventions targeting central symptoms are most commonly used to manage symptom clusters. The aforementioned identification of the central symptoms in a cluster provides a feasible way to formulate interventions based on symptom clusters. Andreas et al. [57] carried out a randomized control trial aimed at alleviating cancer pain through interventions using progressive muscle relaxation (PMR) and analgesia, and the interventions were proven to be useful for other complications. Both the PMR and analgesia interventions significantly improved cancer pain. Cancer survivors reported greater pain improvement and fewer complications through the use of analgesic imagery and PMR than those who did not. This finding showed that controlling the central symptoms could improve multiple other complications.

Simultaneous measures of multiple associated symptoms are another way to manage symptom clusters. A researcher conducted a randomized clinical trial and determined the effectiveness of exercise-based multimodal interventions on treatment-related symptom clusters over time in 42 cancer survivors [58]. Symptom assessments were completed every week while patients were in the hospital and at 3 and 6 months after the therapeutic treatment. Five clusters were determined through principal component analysis, and the changes in the cluster strength scores of the two groups over time were compared by common estimation equations. The intensity of the symptoms in the intervention group decreased significantly with time; however, no decrease was observed in the control group, except for in the emotion cluster. The research provided evidence for useful interventions to reduce the intensity of symptom clusters. Using an invalid questionnaire as a tool to measure symptoms was the main limitation of this research. In addition, the small sample size was also a deficiency of the research, especially because complex statistical methods require a large sample size.

Due to the limited research on symptom cluster management, it is unclear which of the two approaches are more effective for symptom clusters. A multimodal intervention for the symptom cluster might be a new frontier. With the development of symptom cluster management, it is feasible to have other methods to provide treatment for symptom clusters. 


\section{Conclusions}

Research on symptom clusters in cancer survivors is on the rise, whereas the pattern, consistency and intensity of individual symptoms and symptom clusters over time, whether or not they are stable, should be considered. In the future, longitudinal studies are recommended to obtain more in-depth information about the changes in symptom and symptom cluster experience perceived by different groups of cancer survivors.

Due to the unbalanced regional development in China, it is still unknown whether cancer survivors in different regions face the same problems or have the same symptom clusters, so large sample and multicenter studies are urgently needed.

\section{Funding}

This project was supported by the CAMS Innovation Fund for Medical Sciences (2019-I2M-5-046).

\section{Conflicts of Interest}

The author declares no conflicts of interest regarding the publication of this paper.

\section{References}

[1] Lee, L.J., Wehrlen, L., Wallen, G.R., et al. (2020) Symptom Clusters and Influencing Factors in Family Caregivers of Individuals with Cancer. Cancer Nursing. https://doi.org/10.1097/NCC.0000000000000877

[2] Barsevick, A.M., Whitmer, K., Nail, L.M., et al. (2006) Symptom Cluster Research: Conceptual, Design, Measurement, and Analysis Issues. Journal of Pain and Symptom Management, 31, 85-95. https://doi.org/10.1016/j.jpainsymman.2005.05.015

[3] Kwekkeboom, K.L. (2016) Cancer Symptom Cluster Management. Seminars in Oncology Nursing, 32, 373-382. https://doi.org/10.1016/j.soncn.2016.08.004

[4] Chang, V.T., Hwang, S.S., Feuerman, M., et al. (2015) Symptom and Quality of Life Survey of Medical Oncology Patients at a Veterans' Affairs Medical Center: A Role for Symptom Assessment. Cancer, 88, 1175-1183.

https://doi.org/10.1002/(SICI)1097-0142(20000301)88:5<1175::AID-CNCR30>3.0.C $\underline{\mathrm{O} ; 2-\mathrm{N}}$

[5] Lee, K., Oh, E.G., Kim, S., et al. (2019) Symptom Experiences and Health Related Quality of Life among Non-Small Cell Lung Cancer Patients Participating in Clinical Trials. Journal of Clinical Nursing, 28, 2111-2123. https://doi.org/10.1111/jocn.14803

[6] Dodd, M.J., Janson, S., Facione, N., et al. (2001) Advancing the Science of Symptom Management. Journal of Advanced Nursing, 33, 668-676. https://doi.org/10.1046/j.1365-2648.2001.01697.x

[7] Barsevick, A.M. (2007) The Elusive Concept of the Symptom Cluster. Oncology Nursing Forum, 34, 971-980. https://doi.org/10.1188/07.ONF.971-980

[8] Fallowfield, L. (1990) The Quality of Life: The Missing Measurement in Health Care. Souvenir Press, London. 
[9] Hsu, H., Lin, K., Wu, L., et al. (2017) Symptom Cluster Trajectories during Chemotherapy in Breast Cancer Outpatients. Journal of Pain and Symptom Management, 53, 1017-1025. https://doi.org/10.1016/j.jpainsymman.2016.12.354

[10] Dodd, M.J., Miaskowski, C. and Lee, K.A. (2004) Occurrence of Symptom Clusters. Journal of the National Cancer Institute. Monographs, 32, 76-78. https://doi.org/10.1093/jncimonographs/lgh008

[11] Barsevick, A.M. (2007) The Concept of Symptom Cluster. Seminars in Oncology Nursing, 23, 89-98. https://doi.org/10.1016/j.soncn.2007.01.009

[12] Gift, A.G., Stommel, M., Jablonski, A., et al. (2003) A Cluster of Symptoms over Time in Patients with Lung Cancer. Nursing Research, 52, 393-400. https://doi.org/10.1097/00006199-200311000-00007

[13] Fox, S.W. and Lyon, D. (2007) Symptom Clusters and Quality of Life in Survivors of Ovarian Cancer. Cancer Nursing, 30, 354-361. https://doi.org/10.1097/01.NCC.0000290809.61206.ef

[14] Miaskowski, C., Dodd, M. and Lee, K. (2004) Symptom Clusters: The New Frontier in Symptom Management Research. Journal of the National Cancer Institute Monographs, 32, 17-21. https://doi.org/10.1093/jncimonographs/lgh023

[15] Francoeur, R.B. (2005) The Relationship of Cancer Symptom Clusters to Depressive Affect in the Initial Phase of Palliative Radiation. Journal of Pain and Symptom Management, 29, 130-155. https://doi.org/10.1016/j.jpainsymman.2004.04.014

[16] Dodd, M.J., Miaskowski, C. and Paul, S.M. (2001) Symptom Clusters and Their Effect on the Functional Status of Patients with Cancer. Oncology Nursing Forum, 28, 465-470.

[17] Kim, H.J., Barsevick, A.M., Tulman, L., et al. (2008) Treatment-Related Symptom Clusters in Breast Cancer: A Secondary Analysis. Journal of Pain and Symptom Management, 36, 468-479. https://doi.org/10.1016/j.jpainsymman.2007.11.011

[18] Chen, M.L. and Lin, C.C. (2007) Cancer Symptom Clusters: A Validation Study. Journal of Pain and Symptom Management, 34, 590-599. https://doi.org/10.1016/j.jpainsymman.2007.01.008

[19] Bender, C.M., Ergÿn, F.S., Rosenzweig, M.Q., et al. (2005) Symptom Clusters in Breast Cancer across 3 Phases of the Disease. Cancer Nursing, 28, 219-225. https://doi.org/10.1097/00002820-200505000-00011

[20] Walke, L.M., Byers, A.L., Gallo, W.T., et al. (2007) The Association of Symptoms with Health Outcomes in Chronically Ill Adults. Journal of Pain and Symptom Management, 33, 58-66. https://doi.org/10.1016/j.jpainsymman.2006.07.013

[21] Gift, A.G., Jablonski, A., Stommel, M., et al. (2004) Symptom Clusters in Elderly Patients with Lung Cancer. Oncology Nursing Forum, 31, 202-212. https://doi.org/10.1188/04.ONF.203-212

[22] Chen, M.L. and Tseng, H.H. (2005) Identification and Verification of Symptom Clusters in Cancer Patients. The Journal of Supportive Oncology, 3, 28-29.

[23] Fox, S.W., Lyon, D. and Farace, E. (2007) Symptom Clusters in Patients with High-Grade Glioma. Journal of Nursing Scholarship, 39, 61-67.

https://doi.org/10.1111/j.1547-5069.2007.00144.x

[24] Given, B., Given, C., Azzouz, F., et al. (2001) Physical Functioning of Elderly Cancer Patients Prior to Diagnosis and Following Initial Treatment. Nursing Research, 50, 222-232. https://doi.org/10.1097/00006199-200107000-00006

[25] Miaskowski, C. and Lee, K.A. (1999) Pain, Fatigue, and Sleep Disturbances in Oncology Outpatients Receiving Radiation Therapy for Bone Metastasis: A Pilot Study. 
Journal of Pain and Symptom Management, 17, 320-332. https://doi.org/10.1016/S0885-3924(99)00008-1

[26] Dodd, M.J., Cho, M.H., Cooper, B.A., et al. (2010) The Effect of Symptom Clusters on Functional Status and Quality of Life in Women with Breast Cancer. The European Journal of Oncology Nursing, 14, 101-110. https://doi.org/10.1016/j.ejon.2009.09.005

[27] So, W.K., Marsh, G., Ling, W.M., et al. (2009) The Symptom Cluster of Fatigue, Pain, Anxiety, and Depression and the Effect on the Quality of Life of Women Receiving Treatment for Breast Cancer: A Multicenter Study. Oncology Nursing Forum, 36, 205-214. https://doi.org/10.1188/09.ONF.E205-E214

[28] Choi, S. and Ryu, E. (2018) Effects of Symptom Clusters and Depression on the Quality of Life in Patients with Advanced Lung Cancer. European Journal of Cancer Care, 27. https://doi.org/10.1111/ecc.12508

[29] Rebar, A.L., Duncan, M.J., Short, C. and Vandelanotte, C. (2014) Differences in Health-Related Quality of Life between Three Clusters of Physical Activity, Sitting Time, Depression, Anxiety, and Stress. BMC Public Health, 14, 1088.

https://doi.org/10.1186/1471-2458-14-1088

[30] Delaunoit, T., Richard, M.G., Daniel, J.S., et al. (2004) Mortality Associated with Daily Bolus 5-Fluorouracil/Leucovorin Administered in Combination with Either Irinotecan or Oxaliplatin. Cancer, 101, 2170-2176.

https://doi.org/10.1002/cncr.20594

[31] Chow, E., Fan, G., Hadi, S., et al. (2007) Symptom Clusters in Cancer Patients with Bone Metastases. Support Care Cancer, 15, 76-82.

https://doi.org/10.1007/s00520-007-0241-Z

[32] Ferreira, K.A.S.L, Kimura, M., Teixeira, J.M., et al. (2008) Impact of Cancer-Related Symptom Synergisms on Health-Related Quality of Life and Performance Status. Journal of Pain and Symptom Management, 35, 604-616. https://doi.org/10.1016/j.jpainsymman.2007.07.010

[33] Breen, S.J., Baravelli, C.M., Schofield, P.E., et al. (2009) Is Symptom Burden a Predictor of Anxiety and Depression in Patients with Cancer about to Commence Chemotherapy? Medical Journal of Australia, 190, S99-S104.

https://doi.org/10.5694/j.1326-5377.2009.tb02480.x

[34] Brown, V., Sitzia, J., Richardson, A., et al. (2001) The Development of the Chemotherapy Symptom Assessment Scale (C-SAS): A Scale for the Routine Clinical Assessment of the Symptom Experiences of Patients Receiving Cytotoxic Chemotherapy. International Journal of Nursing Studies, 38, 497-510.

https://doi.org/10.1016/S0020-7489(00)00106-1

[35] Chang, V.T., Hwang, S.S., Feuerman, M., et al. (2000) The Memorial Symptom Assessment Scale Short Form (MSAS-SF). Cancer, 89, 1162-1171.

https://doi.org/10.1002/1097-0142(20000901)89:5<1162::AID-CNCR26>3.0.CO;2-Y

[36] Chang, V.T., Hwang, S.S., Kasimis, B., et al. (2004) Shorter Symptom Assessment Instruments: The Condensed Memorial Symptom Assessment Scale (CMSAS). Cancer Investigation, 22, 526-536. https://doi.org/10.1081/CNV-200026487

[37] Kornblith, A.B., Thaler, H.G., Vlamis, V., et al. (1995) Quality of Life of Women with Ovarian Cancer. Gynecologic Oncology, 59, 231-242.

https://doi.org/10.1006/gyno.1995.0014

[38] Seidman, A.D., Portenoy, R., Yao, T.J., et al. (1995) Quality of Life in Phase II Trials: A Study of Methodology and Predictive Value in Patients with Advanced Breast Cancer Treated with Paclitaxel plus Granulocyte Colony-Stimulating Factor. Jour- 
nal of the National Cancer Institute, 87, 1316-1322.

https://doi.org/10.1093/jnci/87.17.1316

[39] Harrison, L.B., Zelefsky, M.J., Pfister, D.G., et al. (1997) Detailed Quality of Life Assessment in Patients Treated with Primary Radiotherapy for Squamous Cell Cancer of the Base of the Tongue. Head Neck, 19, 169-175.

https://doi.org/10.1002/(SICI)1097-0347(199705)19:3<169::AID-HED1>3.0.CO;2-0

[40] Du, P.S., Du, P.A., Polissar, N., et al. (1999) Implementing Guidelines for Cancer Pain Management: Results of a Randomized Controlled Clinical Trial. Journal of Clinical Oncology, 17, 361-370. https://doi.org/10.1200/JCO.1999.17.1.361

[41] Cleel, C.S., Mendoza, T.R., Wang, X.S., et al. (2000) Assessing Symptom Distress in Cancer Patients: The M.D. Anderson Symptom Inventory. Cancer, 89, 1634-1646. https://doi.org/10.1002/1097-0142(20001001)89:7<1634::AID-CNCR29>3.0.CO;2-V

[42] Chen, R.W., Yang, S.L., Xu, Z.Y., et al. (2019) Validation and Application of the Chinese Version of the MD Anderson Symptom Inventory Gastrointestinal Cancer Module (MDASI-GI-C). Journal of Pain and Symptom Management, 57, 820-827. https://doi.org/10.1016/j.jpainsymman.2019.01.007

[43] Hoffman, A.J., Given, B.A., Von, E.A., et al. (2007) Relationships among Pain, Fatigue, Insomnia, and Gender in Persons with Lung Cancer. Oncology Nursing Forum, 34, 785-792. https://doi.org/10.1188/07.ONF.785-792

[44] Liu, L., Fiorentino, L., Natarajan, L., et al. (2009) Pre-Treatment Symptom Cluster in Breast Cancer Patients Is Associated with Worse Sleep, Fatigue and Depression during Chemotherapy. Psycho-Oncology, 18, 187-194.

https://doi.org/10.1002/pon.1412

[45] Reyes-Gibby, C.C., Lu, A.A. anderson, K.O., et al. (2006) Pain, Depression, and Fatigue in Community-Dwelling Adults with and without a History of Cancer. Journal of Pain and Symptom Management, 32, 118-128.

https://doi.org/10.1016/j.jpainsymman.2006.01.008

[46] Beck, S.L., Dudley, W.N. and Barsevick, A. (2005) Pain, Sleep Disturbance, and Fatigue in Patients with Cancer: Using a Mediation Model to Test a Symptom Cluster. Oncology Nursing Forum, 32, 542-543. https://doi.org/10.1188/05.ONF.E48-E55

[47] Aprile, G., Ramoni, M., Dorothy, K., et al. (2008) Application of Distance Matrices to Define Associations between Acute Toxicities in Colorectal Cancer Patients Receiving Chemotherapy. Cancer, 112, 284-292. https://doi.org/10.1002/cncr.23182

[48] Honea, N., Brant, J. and Beck, S.L. (2007) Treatment-Related Symptom Clusters. Seminars in Oncology Nursing, 23, 142-151. https://doi.org/10.1016/j.soncn.2007.01.002

[49] Yun, Y.H., Kim, S.H., Lee, K.M., et al. (2007) Age, Sex, and Comorbidities Were Considered in Comparing Reference Data for Health-Related Quality of Life in the General and Cancer Populations. Journal of Clinical Epidemiology, 60, 1164-1175. https://doi.org/10.1016/i.jclinepi.2006.12.014

[50] Ann, M., Berger, K.P., Parker, S., et al. (2005) Sleep/Wake Disturbances in People with Cancer and Their Caregivers: State of the Science. Oncology Nursing Forum, 32, E98-E126. https://doi.org/10.1188/05.ONF.E98-E126

[51] Maqbali, M.A., Hughes, C., Rankin, J., et al. (2020) Fatigue and Sleep Disturbance in Arabic Cancer Patients after Completion of Therapy: Prevalence, Correlates, and Association with Quality of Life. Cancer Nursing.

[52] Baden, M., Lu, L., Drummond, F.J., et al. (2020) Pain, Fatigue and Depression Symptom Cluster in Survivors of Prostate Cancer. Support Care Cancer, 28, 4813-4824. https://doi.org/10.1007/s00520-019-05268-0 
[53] Whisenant, M., Wong, B., Mitchell, S.A., Beck, S.L. and Mooney, K. (2019) Symptom Trajectories Are Associated with Co-Occurring Symptoms during Chemotherapy for Breast Cancer. Journal of Pain and Symptom Management, 57, 183-189. https://doi.org/10.1016/j.jpainsymman.2018.11.010

[54] Bohlius, J., Tonia, T., Nüesch, E., et al. (2014) Effects of Erythropoiesis-Stimulating Agents on Fatigue- and Anemia-Related Symptoms in Cancer Patients: Systematic Review and Meta-Analyses of Published and Unpublished Data. British Journal of Cancer, 111, 33-45. https://doi.org/10.1038/bjc.2014.171

[55] Okusaka, T. and Furuse, J. (2020) Recent Advances in Chemotherapy for Pancreatic Cancer: Evidence from Japan and Recommendations in Guidelines. Journal of Gastroenterology, 55, 369-382. https://doi.org/10.1007/s00535-020-01666-y

[56] Tiotiu, A., Clerc-Urmès, I. and Martinet, Y. (2015) Efficiency of Erythropoiesis-Stimulating Agents (ESA) in the Treatment of Chemotherapy-Induced Anemia in Patients with Lung Cancer (LC). Nursing Research, 46, 393-400. https://doi.org/10.1183/13993003.congress-2015.PA4842

[57] Andreas, C., Margarita, G., Evaggelos, B., et al. (2016) Guided Imagery and Progressive Muscle Relaxation as a Cluster of Symptoms Management Intervention in $\mathrm{Pa}$ tients Receiving Chemotherapy: A Randomized Control Trial. PLoS ONE, 11, e0156911. https://doi.org/10.1371/journal.pone.0156911

[58] Adamsen, B.L. (2009) The Effect of a Multimodal Intervention on Treatment-Related Symptoms in Patients Undergoing Hematopoietic Stem Cell Transplantation: A Randomized Controlled Trial. Journal of Pain and Symptom Management, 38, 174-190. https://doi.org/10.1016/j.jpainsymman.2008.09.005 